\title{
Performance of DiJKstra, Floyd AND ASTAR ALGORITHMS FOR URBAN TRANSPORT LINES
}

\section{ASTRIT SALLAUKA, RAME LIKAJ}

Abstract: The study of the existing street network built without a layout is very difficult problem (almost insoluble problem). A detailed study needs to be solved a similar problem. It also needs traffic statistics, and various surveys for traffic participants. To make sure the necessary records for an efficient analysis needs a lot of work and a long time to complete. After collecting the necessary data, then through various software such as GIS software, Traffic analysis etc. we can improve the level of services by adding or eliminating any of the problematic routes. The optimization of urban transport lines is a very important problem in bus network planning. Many researchers have been dealing with these phenomena over the years, but there has never been a general pattern applicable to any urban situation as each city is a case in itself and requires more specific approach. In this paper we present the performance of different algorithms for finding shortest route on a network.

Key words: Optimizations of traffic flow, Short path analysis for Urban traffic, Analysis for Urban Traffic, Dijkstra, Floyd, Astar
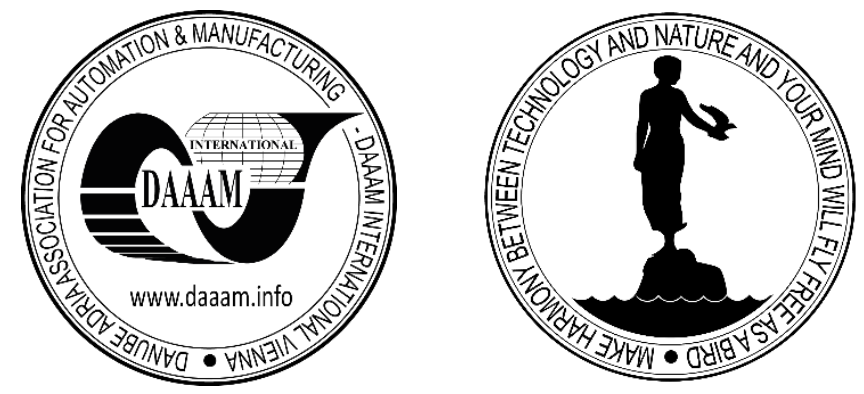

Authors' data: Sallauka A[strit], Likaj R[ame], University of Pristine, Tirana, F.Prime Residence E. D, F.15 10000, Pristine AL, astrit.sallauka@gmail.com, likajrame@gmail.com

This Publication has to be referred as: Sallauka, A[strit] \& Likaj, R[ame] (2019). Performance of Dijkstra, Floyd and Astar Algorithms for Urban Transport Lines, Chapter 21 in DAAAM International Scientific Book 2019, pp.265-270, B. Katalinic (Ed.), Published by DAAAM International, ISBN 978-3-902734-24-2, ISSN 17269687, Vienna, Austria

DOI: $10.2507 /$ daaam.scibook.2019.21 
Sallauka, A. \& Likaj, R.: Performance of Dijkstra, Floyd and Astar Algorithms for ...

\section{Introduction}

The purpose of this paper is to present the performance of different algorithms for finding shortest route of bus lines. Increasing population, the passage of citizens from rural to urban areas, various private and institutional activities in the capital, enormous use of private vehicles to carry out transportation in and out of the city by constantly bypassing public transport are some phenomena that are displaying adverse effects on the environment, time and space. The optimization of urban transport lines is a very important problem in bus network planning. In general, urban transport users face problems in the operation of lines such as: long waiting time at the station, inaccuracy in bus timetables, incompatibility between bus routes and passenger paths and insufficient capacity. These problems pushed citizens to use private transport, which creates unfavourable effects, thus increasing fuel consumption, environmental pollution, traffic intensification, access to public parking spaces and increased accidents. One of the main reasons for the inefficiency of public transport is the fact that bus lines do not cover the network in the best way. An optimization of the bus network is aimed at removing the above-mentioned phenomena by choosing the most reasonable routes related to the frequencies of the movement of citizens.

Different algorithms can be used to plan and optimize urban transport lines, thus solving the problem of urban transport. The logic of these algorithms is based on several objectives, among which the two most important are the minimization of time and mileage taking into account several factors such as: cost, frequency of buses depending on the importance of city areas, respectively taking into account the movements of citizens, coverage of bus lines, time of travel and number of stopovers. In recent years, Pristina has lost the urban structure gained over the years. Because the plans have emphasized only the construction dimension, the urban structural unit of the city has been lost. This has badly affected the estimates and the provision of space for public functions. This also has an adverse effect on the organization of infrastructure and public services.

In addition to the above-mentioned problems, the poor organization of urban traffic lines, respectively the poor coverage of all areas, the lack of an analysis of the movement of citizens in time and space are greatly affecting the over-exploitation of public transport. Such as these have led to private car transport being the most popular mode of transport in the city, thus influencing the large traffic flows, the constant creation of blockage of the main arteries of roads in the capital, and other negative phenomena such as environmental pollution, increased number of accidents, increased travel costs, and so on. According to data obtained, from a total of 477 roads that the municipality of Pristina has, urban traffic covers only 63 of them, or $13 \%$. The average number of stopovers per road is three.

\begin{tabular}{|l|l|}
\hline Number of roads & 477 \\
\hline Roads used by Public Transport & 63 \\
\hline Max stopovers & 10 \\
\hline Min stopovers & 1 \\
\hline Avg. stopovers & 3 \\
\hline
\end{tabular}

Tab. 1. Statistics of stopovers in Prishtina 


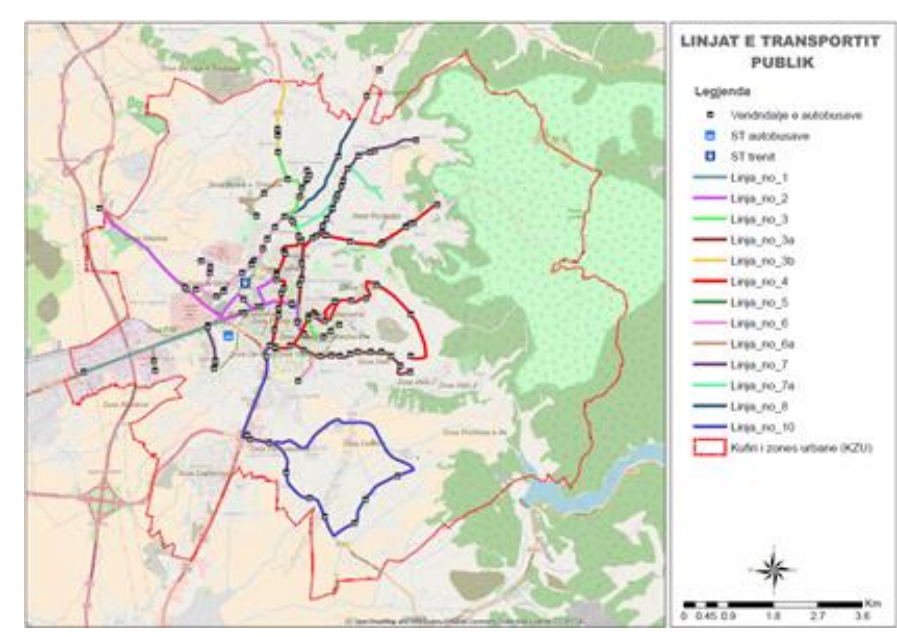

Fig. 1. Existing public transport lines in city of Prishtina

\section{Related work}

There are different approaches for bus lines optimization but there is no general pattern applicable to any urban situation as each city is a case in itself and requires a different approach. The most popular method for finding the shortest path is Dijkstra algorithm. Bozyigit, et al. used a modified version of Dijkstra's algorithm by adding a penalty system, adding penalty costs between vertices [1].

Wang et al. proposed the notion of direct/indirect adjacent edges in weighted directed multigraphs, and used the direct/indirect adjacent edges weighted directed multigraph as a public transportation data model and improved the storage of an adjacency matrix [2]. Yan et al. presented a heuristic algorithm for the public transport network multi-path selection based on the Dijkstra's Algorithm and the Iterative Penalty Method [3]. Ferreira et al. integrated various data sources to one single advisor system which uses Dijkstra Algorithm [4].

\section{Results}

Improving public transport, to reduce the growth of individual motorized traffic, is one of the highlighted measures when it comes to reducing adverse traffic impacts and improving the efficiency of a transport system.

For better and more accurate analysis of urban transport modelling in the city of Prishtina, 77 stopovers have been used within the transport network. Coordinates of these stopovers are taken manually from Open Street Map. From these stopovers, several key aspects were analysed before proposing new lines that would facilitate city traffic and would enable the citizen to reach the destination quickly. First, the time of access to different parts of the city from a point of reference is analysed. Reference points have been determined by some regions where urban transport attendance has been high. 


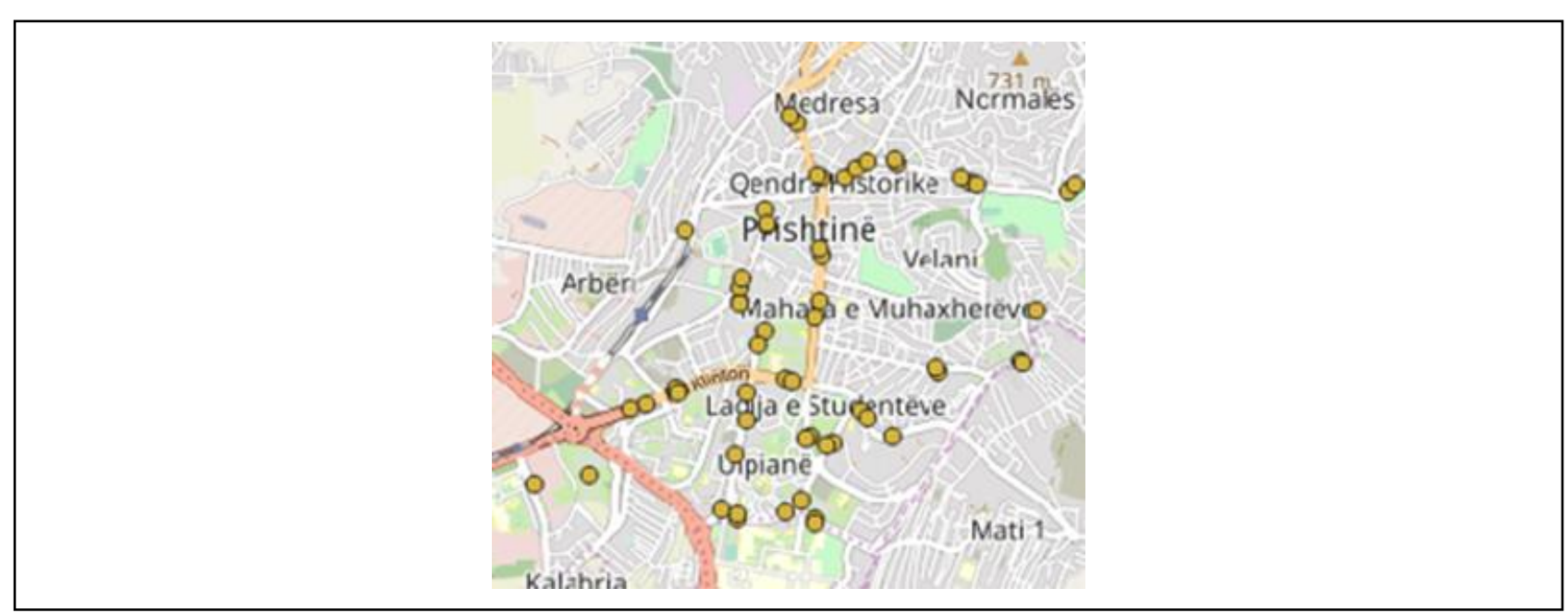

Fig. 2. Stopovers in city of Prishtina

The method of finding the shortest path by passing through as few stopovers has proved to be very efficient. This method takes as parameters the distances between the stopovers and the different algorithms have been used to find the shortest path. The following table shows the stations symbolized by alphabet letters from $\mathbf{A}$ to $\mathbf{O}$ and the distances between these stations.

However, the line establishment only by the shortest distance is not very efficient because it leaves out many residential areas with a population who use public transport for transport to other parts of the city. Therefore, we need to find more effective factors in building the public transport lines. Some of the key criteria analysed are: population access to main roads, access time to the nearest roads, road category, population size by area, use of urban traffic in recent years by area.

For every $n$ stopover we calculate the number of passengers getting in the bus and getting out of the bus:

$$
S=\sum_{i=1}^{n} I_{i}+O_{i}
$$

We normalize the values of distances and passengers served by rescaling the data values between 0 and 1 . The selection of the next stopover can be achieved by calculating the average of stopovers distances $\mathrm{Mi}$ and passenger served by a stopover Ni:

$$
S_{i}=\frac{M_{i}+N_{i}}{2}
$$

According to the data we analyzed, the minimum value of a passenger served at a stopover was 1 and the maximum was 18 passengers. The smallest distance that we got was 212 meters and the longest distance was 918 meters. 


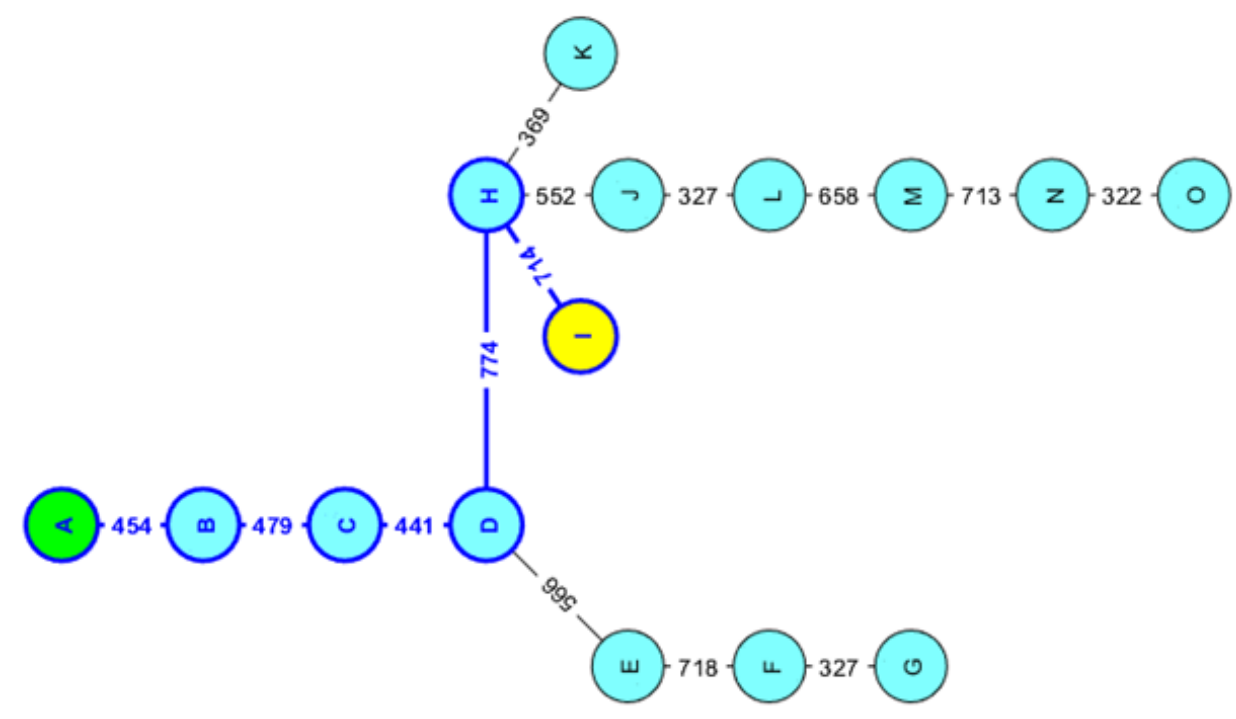

Fig. 3. Finding shortest path based on stopovers distances using Dijkstra's Algorithm

\begin{tabular}{|c|c|c|c|c|}
\hline $\begin{array}{l}\overline{2} \\
\overline{2} \\
\overline{0} \\
\overline{3}\end{array}$ & $\begin{array}{l}\overline{2} \\
\overline{0} \\
\overline{1}\end{array}$ & 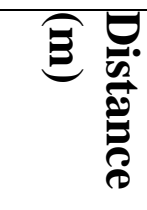 & 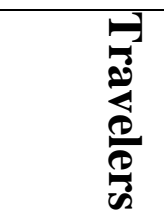 & 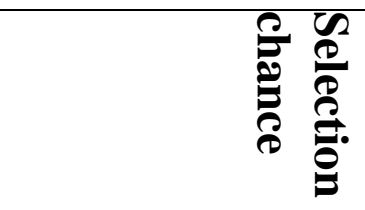 \\
\hline 4436375390 & 1458787667 & 454 & 13 & 0.681553074 \\
\hline 1458787667 & 4434319590 & 479 & 2 & 0.34031828 \\
\hline 4434319590 & 4434334789 & 441 & 5 & 0.455465756 \\
\hline 4434334789 & 4436261491 & 566 & 8 & 0.455174138 \\
\hline 4436261491 & 4436381092 & 718 & 10 & 0.406348942 \\
\hline 4436292791 & 1458787612 & 788 & 5 & 0.209715047 \\
\hline 4436381092 & 4436431589 & 327 & 3 & 0.47737877 \\
\hline 4434334789 & 2070906981 & 774 & 4 & 0.190218297 \\
\hline 2070906981 & 4461986290 & 714 & 9 & 0.379770038 \\
\hline 2070906981 & 4342371169 & 552 & 12 & 0.582736211 \\
\hline 4342371169 & 4469391589 & 327 & 8 & 0.624437594 \\
\hline 4469391589 & 4436274090 & 658 & 6 & 0.331194801 \\
\hline 4436274090 & 4436292791 & 713 & 12 & 0.468713548 \\
\hline 4436292791 & 1458787612 & 322 & 2 & 0.451508082 \\
\hline 2070906981 & 4434334790 & 369 & 3 & 0.447633728 \\
\hline
\end{tabular}

Tab. 2. Results a

Our method is tested on a real-world transport network, in Prishtina, Republic of Kosovo. Using this method some of the bus lines have been modified for best optimization. As a result of our research and studies, with this method we proposed new bus lines for the public transport by making minimal changes to the existing lines and eliminating the overlapping problem of the bus lines, faster arrival at certain destinations and wider coverage of public transport. Our setup is as follows: Intel Core i7-7700K 4.2GHz, 16GB RAM 3200 MHz, Crucial 500GB SSD, GTX 1080 8GB 
Sallauka, A. \& Likaj, R.: Performance of Dijkstra, Floyd and Astar Algorithms for ...

GPU. From the table below we can see that with the increasing number of nodes $\mathrm{A}^{*}$ algorithm performs better in time.

\begin{tabular}{|l|l|l|l|}
\hline & Floyd & Dijkstra & A* \\
\hline 15 nodes & 0.0000158 & 0.0000213 & 0.0000632 \\
\hline 411 nodes & 0.0000769 & 0.0000908 & 0.0001149 \\
\hline
\end{tabular}

Tab. 3. Results b

\section{Conclusion}

The presented method optimizes each line separately, meaning that for each given line there must be sufficient data and it is not possible to provide the algorithm information from the other optimized lines or lines that are being optimized. A bus line can be optimized using different factors such as: the length of the roads, the distance between the stopovers, the line utilization factor, the utilization factor of the given stopover. In this paper we showed the performance of different algorithms for finding best route using distance between stopovers and the utilization factor of stopovers.

\section{References}

Bozyigit, Alican, et al. "Public Transport Route Planning: Modified Dijkstra's Algorithm." 2017 International Conference on Computer Science and Engineering (UBMK), 2017, doi:10.1109/ubmk.2017.8093444.

Wang, Hongmei, et al. "A New Public Transportation Data Model and Shortest-Path Algorithms." 2010 2nd International Asia Conference on Informatics in Control, Automation and Robotics (CAR 2010), 2010, doi:10.1109/car.2010.5456797.

YAN Xiaoyong, NIU Xueqin (Department of Transportation Engineering, Shijiazhuang Railway Institute, Shijiazhuang 050043, China); Study on Heuristic Algorithm for Public Transport Network Multi-Path Selection; Urban Transport of China;2005-03.

Ferreira, Joao C., et al. "Multi-Modal Transportation Advisor System." 2011 IEEE Forum on Integrated and Sustainable Transportation Systems, 2011, doi:10.1109/fists.2011.5973636.

H. Sadrsadat, H. Poorzahedi, A. Haghani, E. Sharifi, "Bus Network Design Using Genetic Algorithm".

A. Sallauka, X. Bajrami, R. Likaj dhe B. Gashi, "Application Genetics Algorithm for finding shortest path in seven cities," International Journal of Civil Engineering \& Technology (IJCIET), volume 9, issue 4, pp. 1516-1526, 2018.

C. f. U. T. Research, «Best Practices in Transit Service Planning, » Florida Department of Transportation, Florida, 2009.

[8] F. Schley, "Urban transport strategy review," Deutsche Gesellschaft für Technische Zusammenarbeit (GTZ) GmbH, 2001.

K. Magzhan, H. M. Jani, "A Review and Evaluations Of Shortest Path Algorithms," international journal of scientific \& technology research, volume 2, issue 6, 2013.

Porkolab, I.,Djambic, G.\& Kucak, D., "Performance Issues and Gains of Caching the Pathfinding Data with FAST-N Algorithm", pp.309-316, 2019 